\title{
Implementation intentions and imagery: individual and combined effects on prospective memory among young adults
}

\author{
Craig McFarland • Elizabeth Glisky
}

Published online: 6 July 2011

(C) Psychonomic Society, Inc. 2011

\begin{abstract}
Prospective memory (PM) has been found to benefit from implementation intentions (i.e., "when I see X, I will do Y"). However, to date, it is unclear whether implementation intentions must incorporate imagery in order to produce a positive effect on PM, or whether the verbal statement alone is sufficient. It is also equivocal whether the use of visual imagery alone improves PM, absent an intentional statement. The present study investigated the individual influences of implementation intentions and imagery, as well as their combined effect, on PM. A total of 64 undergraduates were placed into one of four instructional conditions - read-only, implementation intention, imagery, or combined - and were then tested on a laboratory PM task. The results revealed that participants in the implementation intention, imagery, and combined groups completed significantly more PM tasks than did participants in the read-only group, but they did not differ from one another. Combining implementation intentions and imagery, however, did not improve PM performance over either strategy alone. Additionally, the implementation intention and imagery groups outperformed the read-only group on a secondary ongoing digit detection task. The results of this study suggest that implementation intentions do not require imagery to be effective in improving PM, and that imagery alone has positive effects on PM. Finally, the results of the ongoing digit detection task suggest that the use of implementation intentions and imagery might provide for automatic identification and processing of environmental cues.
\end{abstract}

C. McFarland $(\bowtie) \cdot$ E. Glisky

Department of Psychology, University of Arizona,

Tucson, AZ, USA

e-mail: cpm3@email.arizona.edu
Keywords Prospective memory Implementation intentions $\cdot$ Imagery $\cdot$ Young adults $\cdot$ Strategies

As a forward-looking species, humans have a tendency to develop intentions for future action. In fact, intentions are such an integral part of life that they often color our experiences, particularly on those occasions on which we fail to execute them (e.g., recall the last time you missed, or nearly missed, a scheduled appointment, and the impact it had on your mood). Prospective memory (PM) is a term used to describe the cognitive processes involved in the formation and execution of future intentions, and is described as "remembering to remember." Although we are often able to successfully execute PM tasks, we frequently fail to do so, despite the benefit of motivation that the sometimes dire consequences of PM failure can provide (e.g., forgetting to call your mother on her birthday). In this study, we investigated the effect of strategies designed to improve PM performance.

Several factors have been shown to affect PM performance. Cue saliency, or the degree to which a target item stands out relative to other, nontarget items, impacts upon PM performance, with more highly salient cues typically producing greater PM performance (Einstein \& McDaniel, 1990; McDaniel \& Einstein, 1993; but see McDaniel, Glisky, Rubin, Guynn, \& Routhieaux, 1999). For example, Einstein and McDaniel reported improved PM performance when the targets were unfamiliar words (e.g., sone) relative to familiar words (e.g., rake). The authors suggested that those results were likely due to the fact that the uncommon words were more distinctive, and therefore stood out against a backdrop of otherwise familiar terms. McDaniel and Einstein (2000) contended that salient items involuntarily capture attention, resulting 
in an evaluation of the item's significance to ongoing and subsequent future activity.

A second factor affecting PM performance is the strength of the association between a target and an intended action. McDaniel, Guynn, Einstein, and Breneiser (2004) reported significantly improved PM performance under conditions in which cue and intention were strongly associated. In that study, participants were presented with word pairs and were asked to write the second word if they ever saw the first word of the pair during the completion of a word-rating task. PM performance was greater when words were highly associated (e.g., spaghetti-sauce) relative to conditions of weaker association (e.g., spaghetti-needle), and these results were interpreted in the context of an automatic associative memory process. Such an interpretation rests on the notion that once a strong association between cue and intention has been created, subsequent encounters with the cue will result in obligatory, reflexive retrieval of the associated intention, requiring little effortful retrieval, as long as the cue was fully processed (Moscovitch, 1994).

The impact that a strong association between target and intention can have on PM is a factor believed to underlie the effectiveness of one of the more extensively studied PM strategies. Gollwitzer (1999) developed a technique to improve PM that integrates two critical components of future intentions: the future situation that may provide an opportunity to execute one's intention, including "where" and "when," and the intended action, or the "what." This technique comprises the development of an association in the form of "if X occurs, then I will do Y," and it has been dubbed an implementation intention. Implementation intentions have been the focus of a growing body of empirical research over the last 10 years, the majority of which have suggested that their use is an effective means of fulfilling future commitments (Chasteen, Park, \& Schwarz, 2001; Cohen \& Gollwitzer, 2008; Kardiasmenos, Clawson, Wilken, \& Wallin, 2008; Liu \& Park, 2004; McDaniel, Howard, \& Butler, 2008; Milne, Orbell, \& Sheeran, 2002; Orbell, Hodgkins, \& Sheeran, 1997; but see Einstein, McDaniel, Williford, Pagan, \& Dismukes, 2003; Schnitzspahn \& Kliegel, 2009).

Although considerable research supports the view that implementation intentions are an effective means of improving PM, several questions regarding the mechanisms that constitute their effects remain unanswered. Importantly, it remains unclear which features of an implementation intention are necessary and sufficient for future PM success. The present study seeks to address this issue.

In Gollwitzer's (1999) initial formulation, an implementation intention was described as a verbal commitment specified in terms of an "if . . . then" statement, and according to him, the pairing of a future context conducive to task completion with a specific course of action to be completed in that context results in successful PM. In other words, the pairing of cue and intention results in a strong association between the two, and subsequently yields automatic triggering of an intention when the cue is encountered. That Gollwitzer framed the verbal "if . . . then" component of implementation intentions as a commitment may be important, and this has led others to argue that implementation intentions are effective because they foster the development of a personal commitment to action (Ellis \& Freeman, 2008). One possible mechanism of increased commitment is that the PM task itself is imbued with greater significance or importance, which has been shown to improve PM (Kliegel, Martin, McDaniel, \& Einstein, 2001, 2004).

Several studies have augmented the development of a verbal statement prescribed by Gollwitzer (1999) with a period of imagining. In fact, the majority of recent investigations into the effectiveness of implementation intentions on PM have incorporated imagery (Kardiasmenos et al., 2008; Liu \& Park, 2004; McDaniel et al. 2008; McDaniel \& Scullin, 2010; Meeks \& Marsh, 2010; Schnitzspahn \& Kliegel, 2009), despite findings suggesting that the inclusion of imagery is not necessary to improve PM (Cohen \& Gollwitzer, 2008; Gollwitzer \& Brandstätter, 1997). Though studies incorporating imagery with the verbal component of implementation intention instructions have produced positive effects on PM, the benefit of imagery is unclear, because the value of adding imagery to a verbal "if . . then" statement has not been explored. Thus, one goal of the present study is to investigate whether the inclusion of imagery in an implementation intention instruction provides any additive benefit, over that of implementation intention instructions comprising solely an "if . . . then" statement.

A second goal of the present study is to investigate whether imagery alone, without accompanying implementation intention instructions, provides for improved PM. The few studies that have investigated the effect of imagery on PM have produced inconsistent findings. Guynn, McDaniel, and Einstein (1998) reported findings in which a group of participants that received a reminder of the PM task along with imagery instructions outperformed a group that received a reminder but no imagery instructions. However, the imagery group did not outperform a group that received what could be considered standard PM instructions. Similarly, McDaniel et al. (2008) reported no benefit of imagery instruction on PM performance, but did find that imagery plus a verbal "if . . . then" statement resulted in improved PM performance, suggesting that the verbal statement, not imagery, is the critical agent. However, Meeks and Marsh (2010) reported improved PM performance in an imagery-only condition over that of standard instruction. Furthermore, the benefit of the imagery-only instruction was commensurate with that of a 
condition in which imagery was combined with a verbal "if . . . then" statement. Overall, the ability of imagery instruction alone to improve PM performance is equivocal, with the few studies that have explored the issue having produced inconsistent findings.

Thus, although implementation intentions have been shown to be effective in improving PM, what is required of an implementation intention is unclear. Several studies have demonstrated that implementation intention instructions of the sort first put forth by Gollwitzer (1999), namely those relying on the formation of an "if . . . then" statement, are an effective means of successfully completing future intentions. Also, it is clear that the inclusion of imagery along with a verbal statement enhances PM performance, or at least does not have the effect of degrading performance relative to an "if . . . then"-only instruction. What is not clear is whether the addition of imagery to a verbal statement provides any additive benefit over recitation of an "if . . . then" statement. Additionally, whether imagery alone can effectively improve PM remains unclear. The present study was designed to address these two issues.

\section{Method}

\section{Participants}

Sixty-four young adults were recruited from undergraduate psychology courses. Students were awarded class credit for participating in the experiment.

\section{Materials}

The ongoing background task in this experiment consisted of a multiple-choice test of general knowledge and trivia. A total of 196 questions were selected from the McDaniel et al. (1999) study and were presented on a Gateway E-6610D PC, using DMDX (Forster \& Forster, 2003). Questions appeared in the center of the monitor. Beneath each question were four response options, corresponding to the multiple-choice answers. Participants indicated their answer choice by pressing one of four keys on the computer keyboard (labeled "A," "B," "C," or "D") and were allowed a maximum of $12 \mathrm{~s}$ to respond to each question. Successive questions appeared at 12-s intervals, regardless of speed of responding. Questions were presented in a different random order for each participant. Included among the multiplechoice questions were 8 target questions about the United States in which the word "state" appeared. Those questions appeared at approximately 5-min intervals throughout the course of the experiment. In addition to the ongoing triviaquestion task, participants were engaged in an auditory detection task in which digits (1-9) were presented at the rate of one every $2 \mathrm{~s}$ through speakers attached to the computer. Participants were instructed to press a mouse key whenever they heard two consecutive odd digits. There were 55 instances of consecutive odd digits. PM targets were always presented at least $15 \mathrm{~s}$ after the presentation of consecutive odd digits. The auditory digit detection task was included in an attempt to make the overall task requirements more demanding. This was done so as to prevent performance under the standard instruction from being too high to discern any potential benefit of strategy use.

\section{Procedure}

The experiment lasted approximately $1 \mathrm{~h}$. After providing informed consent, participants were given a sheet of paper detailing task instructions and were asked to read it carefully. The instructions indicated that as participants in the study, they would be asked to complete a multiplechoice test of general knowledge and trivia, and that they would have a total of $12 \mathrm{~s}$ to respond to each question. They were also told that they would need to complete a secondary task (i.e., the PM task), and at that time were instructed to direct their attention to the computer monitor, on which additional instructions were presented. At that point, the instructions differed depending on the condition into which a participant was placed.

Participants were placed into one of four conditions: read-only, implementation intention, imagery, or combined. In the read-only condition, participants read silently from the computer screen a PM instruction consisting of the statement 'If you see questions pertaining to 'states,' you should press the ' 6 ' key." They were then asked to write that exact instruction one time on a provided sheet of paper and to repeat it once aloud to the experimenter. In the implementation intention condition, participants silently read the read-only instruction from the computer screen, followed by a more specific implementation intention ('When I see the word 'state,' I will press the ' 6 ' key."), which they also read silently from the computer. They were then asked to write the exact implementation intention on a sheet of paper and to repeat it once aloud to the experimenter. Participants in the imagery condition silently read the read-only instruction and were then asked to imagine themselves seeing questions about "states" and to picture themselves pressing the " 6 " key in response to those questions. Participants were given $30 \mathrm{~s}$ to imagine, and they were told to continue imagining the scenario for the entire $30 \mathrm{~s}$, even if the image came to them much more quickly. Participants in the combined condition silently read the read-only instruction and were then asked to imagine themselves seeing questions about "states" and to picture themselves pressing the "6" key in response to those 
questions. Participants were given $15 \mathrm{~s}$ to imagine and were told to continue imagining the scenario for the entire $15 \mathrm{~s}$, even if the image came to them much more quickly. After imagining, participants silently read the implementation intention and were asked to write it verbatim one time and then to repeat it aloud to the experimenter.

Following the presentation of instructions, participants completed a series of five practice questions so that they could become familiar with the types of questions they would encounter during the experiment. Included among the practice questions was one containing the word "state." In instances in which a participant failed to press the " 6 " key in response to that practice question, the participant was informed of the omission, and the practice questions were presented again, until the participant correctly pressed the " 6 " key when presented with a question containing the word "state." Next, participants were given the opportunity to practice the digit detection task. A series of 12 digits was presented to each participant, at the rate of one digit every $2 \mathrm{~s}$.

After completion of the practice trials, no further mention of the PM task was made. The multiple-choice trivia questions with the embedded PM task, along with the auditory digit detection task, then proceeded for $42 \mathrm{~min}$, after which participants were asked to describe the tasks involved (i.e., answering multiple-choice questions, pressing the " 6 " key when seeing the word "state"). At that time, participants completed a brief recognition memory test as an additional measure of retrospective memory. These measures were included in an attempt to ensure that any PM performance differences were not due to group differences in retrospective memory. The recognition memory test took approximately $12 \mathrm{~min}$ to complete and consisted of a study phase in which 56 words were presented visually on the computer at a rate of approximately one word every $2 \mathrm{~s}$. Immediately following completion of the study phase, participants completed the test phase, which consisted of visual presentation of 112 words, half previously studied, half new. For each word, participants were asked to press a key labeled "yes" if they believed that they had seen the word in the previous study phase, and to press a key labeled "no" if they believed that they had not seen it in the previous study phase. Following completion of the recognition test, participants were debriefed regarding the purpose of the study and were provided time to ask any questions they might have had.

\section{Results}

Prospective memory task

For each participant, the proportion of correct PM responses was computed. Correct PM responses were defined as a pressing of the " 6 " key made in response to the presentation of questions in which the word "state" appeared, with the keypress occurring either during the presentation of the target question or immediately following its presentation, but prior to the presentation of the next trivia question. A value of 1 was given to "6" keypresses made prior to the presentation of succeeding questions, and a value of 0 was given for omissions or for keypresses made following the presentation of subsequent questions. Table 1 presents the mean proportions of correct responses as a function of instructional condition. These data were submitted to a one-way ANOVA, which revealed a significant effect of instructional condition, $F(3,60)=3.70$, $M S E=17.27, p<.05$. Planned comparisons using least significance tests revealed that, as expected, participants in the implementation intention condition $(M=.54, S D=.23)$ completed significantly more PM tasks than did participants in the read-only condition $(M=.30, S D=.30), t(30)=2.46$, $p<.05$. Participants in the imagery condition $(M=.55$, $S D=.29$ ) also completed significantly more PM tasks than did those in the read-only condition, $t(30)=2.54, p<.05$. Finally, participants in the combined condition $(M=.59$, $S D=.59$ ) also outperformed the participants in the read-only condition, $t(30)=3.01, p<.01$. The three experimental groups, however, did not differ significantly from one another (all $t \mathrm{~s}<1$ ). The inclusion of "late" responses made during the presentation of questions immediately following the target question did not alter this pattern.

\section{Ongoing-task performance}

The number of trivia questions answered correctly was then analyzed as a measure of ongoing-task performance. Mean proportions of trivia questions answered correctly are presented in Table 2 as a function of instructional condition. A one-way ANOVA revealed no group differences in terms of the number of trivia questions answered correctly, $F<1$, n.s. There were also no group differences in the time taken to respond to trivia questions, $F<1$, n.s.

Performance of the auditory digit detection task was also analyzed, and the data were submitted to a one-way ANOVA. Mean digit detection performance is shown in Table 3 as a function of instructional condition. The results

Table 1 Mean $(S D)$ proportions of PM tasks executed as a function of instructional group

Instructional Group

\begin{tabular}{ll}
\hline Read-only & $.31(.30)$ \\
Implementation intention & $.54(.23)$ \\
Imagery & $.55(.29)$ \\
Combined & $.59(.29)$ \\
\hline
\end{tabular}


Table 2 Mean $(S D)$ proportions of trivia questions correctly answered as a function of instructional group

\begin{tabular}{ll} 
Instructional Group & \\
\hline Read-only & $.40(.08)$ \\
Implementation intention & $.38(.07)$ \\
Imagery & $.38(.05)$ \\
Combined & $.41(.04)$ \\
\hline
\end{tabular}

indicated a main effect of instructional condition on digit detection performance, $F(3,60)=4.43, M S E=.166, p<.01$. Planned comparisons using least significant difference tests revealed that participants in the implementation intention condition $(M=.59, S D=.21)$ significantly outperformed participants in the read-only condition $(M=.35$, $S D=.21), t(30)=3.46, p<.01$. Participants who received imagery instructions $(M=.52, S D=.18)$ also outperformed participants in the read-only condition, $t(30)=2.50, p<.05$. However, the performance of participants in the combined condition $(M=.44, S D=.17)$ did not differ from that of participants in the read-only condition, $t(30)=1.33$, n.s. Comparisons made between the implementation intention and imagery conditions indicated that the digit detection performance of the two groups did not significantly differ, $t<1$. Similarly, the performance of participants in the imagery condition did not differ from that of participants in the combined condition, $t(30)=-1.13$, n.s. However, the performance of participants in the implementation intentions condition was significantly greater than that of participants in the combined condition, $t(30)=-2.13, p<.05$.

\section{Retrospective memory}

Two measures were included to assess retrospective memory. A recognition memory test was completed by all participants following the completion of the PM task. A one-way ANOVA revealed that there were no group differences in recognition memory performance, $F(3,60)=1.64, p>.05$, since the hit rates were very similar between the implementation intention $(M=.79, S D=.11)$, imagery $(M=.77, S D=.09)$, combined $(M=.80, S D=.11)$, and read-only $(M=.76, S D=.10)$ groups. Additionally, false alarm rates were similar between

Table 3 Mean $(S D)$ proportions of digits detected as a function of instructional group

Instructional Group

Read-only

$.35(.21)$

Implementation intention

$.59(.21)$

Imagery

$.52(.18)$

Combined

$.44(.17)$ the implementation intention $(M=.08, S D=.06)$, imagery $(M=.11, S D=.10)$, combined $(M=.10, S D=.08)$, and read-only $(M=.08, S D=.05)$ groups.

Following completion of the recognition memory test, participants were asked to describe the tasks involved in the study, providing a second measure of retrospective memory. All participants were able to successfully recall the requirement to press the " 6 " key whenever the word "state" appeared during the trivia question task. As expected, we found no differences in retrospective memory between the four instructional conditions. Therefore, retrospective memory is unlikely to have contributed to group performance differences on the PM or auditory digit detection tasks.

\section{Discussion}

In the present study, three separate but related strategies improved PM performance over performance following standard read-only instructions. These results add to those of the few studies that have found positive effects of implementation intentions among young adults. However, this is the first study of which we are aware that has sought to investigate and compare the specific contributions to PM performance of the verbal "if . . . then" statement of an implementation intention and of visual imagery, as well as the effects of the combination of these strategies.

Although implementation intention instructions that incorporate imagery have been shown to improve PM, the role that imagery plays in improving PM remains unclear, because previous studies that employed only an "if . . . then" statement also reported improved PM. Furthermore, in the few studies that have investigated the role of imagery alone, the results have been equivocal as to its effectiveness (McDaniel et al., 2008; Meeks \& Marsh, 2010).

The results of the present study provide some insight into the above questions. First, implementation intention instructions that included only a verbal "if ... then" statement improved PM performance as compared to standard PM instructions, consistent with previous work (Cohen \& Gollwitzer, 2008; Gollwitzer \& Brandstätter, 1997). This finding suggests that implementation intention instructions do not require the inclusion of visual imagery to be effective. Second, participants in the present study who received imagery-only instructions outperformed participants in the standard instruction group. The present study is only the second of which we are aware that has found imagery-only instructions to improve PM performance over that obtained with standard instructions (Meeks \& Marsh, 2010), and thus the study contributes to our understanding of the effectiveness of imagery in improving PM. The results of the present study are also consistent with previous reports of improved PM following the pairing of a 
verbal "if . . . then" statement with visual imagery (Kardiasmenos et al., 2008; Liu \& Park, 2004; McDaniel et al., 2008; McDaniel \& Scullin, 2010; Meeks \& Marsh, 2010). Interestingly, however, pairing the "if . . . then" statement with imagery did not result in PM performance greater than that produced by either the "if . . . then" statement or imagery alone. Taken together, these results suggest that both implementation intentions and imagery, in isolation, are sufficient to improve PM, but that their integration provides no additional benefit over either strategy alone.

Several theories exist as to what provides for the improved PM performance typically seen following implementation intention instructions. One idea is that a cue takes on greater saliency following the formation of an implementation intention. Along these lines, Gollwitzer (1999) contended that by specifying the future context in which a cue may be encountered, implementation intentions result in a heightened awareness of, or accessibility to, environmental cues. This idea resonates with the notion that once an intention is formed, the situational cue to which one is supposed to respond resides in a heightened state of activation (Goschke \& Kuhl, 1993, 1996). It is possible that the improved PM performance seen in the implementation intention group was due, at least in part, to this increased cue saliency. Although cue saliency was not manipulated in the present study, an increase in cue saliency could be expected to reduce the resources required to identify PM task-relevant cues (e.g., requiring less monitoring), allowing for additional resources to be devoted to the ongoing trivia and digit detection tasks. Consistent with this notion, participants in the implementation intention group outperformed participants in the read-only condition on the auditory digit detection task. The improved PM performance following implementation intention instructions in the present study might also have been due to the creation of a strong association between cue and intention. Gollwitzer (1999) argued that one product of phrasing one's future intention in the form of an "if . . then" statement is the development of an enhanced link between a cue and a previously formed intention. This enhanced association between cue and intention might then provide for automatic processing of a subsequently presented cue and reflexive associative retrieval of the related intention. One line of evidence often presented in support of this notion pertains to ongoing-task performance.

The results of several studies have indicated that ongoing-task performance suffers when participants are required to simultaneously complete a PM task, relative to participants who complete an ongoing task in isolation - an effect referred to as task interference - which may reflect the resource-demanding nature of PM tasks. Investigations of implementation intentions, however, have revealed that the ongoing-task performance of participants in implemen- tation intention conditions is often significantly greater than that of participants in standard PM instruction conditions (Cohen \& Gollwitzer, 2008). Combined with improved PM performance, these results have been interpreted as evidence that implementation intentions produce a strong association between cue and intention (cf. Meeks \& Marsh, 2010), such that the PM task itself becomes less resourcedemanding, thereby freeing up attentional resources to be applied elsewhere (i.e., to the ongoing task). The pattern of results in the present study in which implementation intention instructions improved not only PM performance, but also ongoing-task performance, is consistent with previous findings and fits with the theory that implementation intentions provide for automatic, reflexive retrieval of intentions in the presence of PM cues. It is important to point out, however, that although implementation intentions have been thought to result in obligatory, reflexive responding in the presence of cues (Gollwitzer, 1999; McDaniel et al., 2004), recent evidence has suggested that retrieval of the intention may be effortless, but execution of the intention likely requires additional cognitive processes (McDaniel \& Scullin, 2010).

Although the discussion thus far has centered on the possible mechanisms underlying the effect of implementation intentions on PM performance, the same arguments could be made for the effect of imagery on PM performance. For instance, imagining the future context in which an intention is to be executed may make PM cues more salient, as a result of heightened activation following their imagining.

The use of imagery in the present study could also have resulted in the development of a strong association between cue and intention, as has been suggested regarding the effect of implementation intentions. Having been instructed to both imagine themselves seeing the target word ("state") appear on the computer screen and responding by pressing the " 6 " key, participants might have linked the cue and intention in a manner not done by participants in the readonly condition. This linkage of cue and intention could then have resulted in rapid retrieval of the previously developed intention once the cue was encountered. Such an argument also fits with the imagery group's performance of the ongoing task, in which they also outperformed the read-only group, suggesting that the use of imagery has the effect of reducing the resources required to complete the PM task.

That both implementation intentions and imagery may rely on the same or similar mechanisms is also supported, at least in part, by the performance of the combined group. More specifically, had the two strategies relied on different mechanisms, employing them in tandem might have been expected to provide an added benefit over either strategy alone, in essence providing the best of both strategies. Because this did not occur, it is possible that the two strategies share the same mechanisms. 
The pattern of results for the combined group was a bit more complex than that of either the implementation intention or the imagery group, however. Although the PM performance of the combined group was equivalent to that of both implementation intention and imagery groups, combined instructions did not confer the anticipated advantage in completion of the ongoing digit detection task. Instead, the performance of the combined group did not differ from that of the read-only or imagery groups, but was significantly lower than that of the implementation intention group. This pattern suggests that participants in the combined group might have approached the task somewhat differently than did participants in either the implementation intention or imagery groups.

It is possible that by providing participants in the combined group with two distinct forms of instruction (i.e., implementation intention and imagery), the examiner unwittingly increased the perceived importance of the PM task, such that PM performance increased over that seen in the read-only group, but ongoing-task performance suffered in comparison to that produced by the implementation intention group (their performance did not significantly differ from that of the imagery group). This pattern is consistent with recent work revealing that greater perceived task importance improves PM performance, albeit at the expense of ongoing-task performance (Kliegel et al., 2001, 2004).

In summary, the present study contributes to our understanding of implementation intentions in several ways. First, it is clear that implementation intentions need not incorporate imagery in order to produce positive effects on PM performance, consistent with earlier findings of implementation intentions. Also consistent with previous findings, the present pattern of results (i.e., improved PM and auditory digit detection performance relative to a readonly group) for the implementation intention group supports the notion that implementation intentions may result in increased cue saliency and or strengthened cue-intention associations. Second, the present results suggest that the use of imagery alone is sufficient to enhance PM performance. Although the present study was not designed to investigate potential mechanisms underlying the effectiveness of imagery, the similar patterns of performance between the imagery implementation intention groups may be indicative of similar processes across the two strategies. Future studies should further explore this possibility. Finally, the combination of "if . . . then" implementation intention instructions with imagery conferred no benefit over and above that seen for either strategy alone.

Although the results of the present study suggest that verbal "if . . . then" implementation intentions and imagery produce similar effects on PM performance, there may be occasions on which the use of one strategy is preferable to the other. For instance, the use of imagery may be better suited for some situations that are easy to imagine or for "if ... then" statements that can be further enriched through the use of visual imagery. Future work should examine whether individual characteristics of people or situations impact the effectiveness of a particular strategy on PM performance.

\section{References}

Chasteen, A. L., Park, D. C., \& Schwarz, N. (2001). Implementation intentions and facilitation of prospective memory. Psychological Science, 12, 457-461.

Cohen, A.-L., \& Gollwitzer, P. M. (2008). The cost of remembering to remember: Cognitive load and implementation intentions influence ongoing task performance. In M. Kliegel, M. A. McDaniel, \& G. O. Einstein (Eds.), Prospective memory: Cognitive, neuroscience, developmental, and applied perspectives (pp. 367-390). Mahwah, NJ: Erlbaum.

Einstein, G. O., \& McDaniel, M. A. (1990). Normal aging and prospective memory. Journal of Experimental Psychology. Learning, Memory, and Cognition, 16, 717-726.

Einstein, G. O., McDaniel, M. A., Williford, C. L., Pagan, J. L., \& Dismukes, R. K. (2003). Forgetting of intentions in demanding situations is rapid. Journal of Experimental Psychology. Applied, 9, 147-162. doi:10.1037/1076-898X.9.3.147.

Ellis, J. A., \& Freeman, J. E. (2008). Ten years on: Realizing delayed intentions. In M. Kliegel, M. A. McDaniel, \& G. O. Einstein (Eds.), Prospective memory: Cognitive, neuroscience, developmental, and applied perspectives (pp. 1-27). Mahwah, NJ: Erlbaum.

Forster, K. I., \& Forster, J. C. (2003). DMDX: a windows display program with millisecond accuracy. Behavior Research Methods, Instruments, \& Computers, 35, 116-124.

Gollwitzer, P. M. (1999). Implementation intentions: strong effects of simple plans. American Psychologist, 54, 493-503.

Gollwitzer, P. M., \& Brandstätter, V. (1997). Implementation intentions and effective goal pursuit. Journal of Personality and Social Psychology, 73, 186-199. doi:10.1037/0022-3514.73.1.186.

Goschke, T., \& Kuhl, J. (1993). Representation of intentions: persisting activation in memory. Journal of Experimental Psychology. Learning, Memory, and Cognition, 19, 1211-1226. doi: $10.1037 / 0278-7393.19 .5 .1211$.

Goshke, T., \& Kuhl, J. (1996). Remembering what to do: Explicit and implicit memory for intentions. In M. A. Brandimonte, G. O. Einstein, \& M. A. McDaniel (Eds.), Prospective memory: Theory and applications (pp. 53-91). Mahwah, NJ: Erlbaum.

Guynn, M. J., McDaniel, M. A., \& Einstein, G. O. (1998). Prospective memory: when reminders fail. Memory \& Cognition, 26, 287298.

Kardiasmenos, K. S., Clawson, D. M., Wilken, J. A., \& Wallin, M. T. (2008). Prospective memory and the efficacy of a memory strategy in multiple sclerosis. Neuropsychology, 22, 746-754. doi:10.1037/a0013211.

Kliegel, M., Martin, M., McDaniel, M. A., \& Einstein, G. O. (2001). Varying the importance of a prospective memory task: differential effects across time- and event-based prospective memory. Memory, 9, 1-11. doi:10.1080/09658210042000003.

Kliegel, M., Martin, M., McDaniel, M. A., \& Einstein, G. O. (2004). Importance effects on performance in event-based prospective memory tasks. Memory, 12, 553-561. doi:10.1080/ 09658210344000099 . 
Liu, L. L., \& Park, D. C. (2004). Aging and medical adherence: the use of automatic processes to achieve effortful things. Psychology and Aging, 19, 318-325. doi:10.1037/0882-7974.19.2.318.

McDaniel, M. A., \& Einstein, G. O. (1993). The importance of cue familiarity and cue distinctiveness in prospective memory. Memory, 1, 23-41.

McDaniel, M. A., \& Einstein, G. O. (2000). Strategic and automatic processes in prospective memory retrieval: a multiprocess framework. Applied Cognitive Psychology, 14, S127-S144. doi:10.1002/acp.775.

McDaniel, M. A., Glisky, E. L., Rubin, S. R., Guynn, M. J., \& Routhieaux, B. C. (1999). Prospective memory: a neuropsychological study. Neuropsychology, 13, 103-110.

McDaniel, M. A., Guynn, M. J., Einstein, G. O., \& Breneiser, J. (2004). Cue-focused and reflexive-associative processes in prospective memory retrieval. Journal of Experimental Psychology. Learning, Memory, and Cognition, 30, 605-614.

McDaniel, M. A., Howard, D. C., \& Butler, K. M. (2008). Implementation intentions facilitate prospective memory under high attention demands. Memory \& Cognition, 36, 716-724. doi:10.3758/MC.36.4.716.
McDaniel, M. A., \& Scullin, M. K. (2010). Implementation intention encoding does not automatize prospective memory responding. Memory \& Cognition, 38, 221-232.

Meeks, J. T., \& Marsh, R. L. (2010). Implementation intentions about nonfocal event-based prospective memory tasks. Psychological Research, 74, 82-89. doi:10.1007/s00426-008-0223-x.

Milne, S., Orbell, S., \& Sheeran, P. (2002). Combining motivational and volitional interventions to promote exercise participation: protection motivation theory and implementation intentions. British Journal of Health Psychology, 7, 163-184.

Moscovitch, M. (1994). Memory and working with memory: Evaluation of a component process model and comparison with other models. In D. L. Schacter \& E. Tulving (Eds.), Memory systems 1994 (pp. 269-310). Cambridge, MA: MIT Press.

Orbell, S., Hodgkins, S., \& Sheeran, P. (1997). Implementation intentions and the theory of planned behavior. Personality and Social Psychology Bulletin, 23, 945-954.

Schnitzspahn, K. M., \& Kliegel, M. (2009). Age effects in prospective memory performance within older adults: the paradoxical impact of implementation intentions. European Journal of Ageing, 6, 147-155. doi:10.1007/s10433-009-0116-x. 